\title{
THE BIOLOGICAL PROPERTIES OF CANCER STEM CELLS IN MALIGNANT GLIOMAS IN TISSUE CULTURE: A REVIEW
}

\section{ABSTRACT}

This review presents recent data on the biological properties of cancer stem cells in brain gliomas. The possibilities of using of experimental methods of cell culture and immunohistochemical identification of tumor stem cells as a part of neurospheres (tumorospheres) are shown. Methodological features of neurospheres culture are described.

Literature data about the dependence of neurospheres formation activity in the culture on the degree of anaplasia of original glioma tissues are presented considering the disease outcomes in neuro-oncological patients. Possibilities of the practical use of cultured tumorospheres in the study of selective impact of antiblastic drugs on tumor stem cells in gliomas are described.

KEYWORDS: cancer stem cells, glioma, cell culture, neurosphere

To date, there has been accumulated a large amount of data, suggesting that malignant glial brain tumors form a cell hierarchy of undifferentiated progenitor cells like normal nerve tissue [1]. In literature this self-renewing population of cells with tumorigenic properties is defined as cancer stem cells (CSCs), which closely resemble neural stem cells (NSCs) from neurogenerating areas of the normal brain [2, 3]. Like NSCs glioma CSCs are able to self-replicate and express markers of primary neurons and gliocytes - astrocytes and oligodendrocytes [4-6]. However, unlike normal NSCs, CSCs function in an uncontrolled manner and cause further growth of the tumor after its primary resection.

It is considered that CSCs are an important step in the initiation, maintenance and progression of primary and further growth of partially removed brain gliomas, while the significance of the tumor microenvironment is not less important in these processes. Herewith the content of CSCs in glioma tissue increases in proportion to the increase of the malignancy degree with a parallel increase in the expression levels of immunocytochemical markers of their stem properties [4, 7-9]. It is generally recognized that the identification of CSCs in various tumor types, as well as in brain gliomas is associated with a poor prognosis of the disease [10-14].

The use of the principles accepted in the study of normal NSCs to the study of CSCs populations in brain tumors establishes a link between normal neurogenesis and tumorigenesis in the brain. In this case identification of CSCs in brain tissue provides a powerful tool for the study of tumorigenicity process in the CNS, as well as for the development of optimized treatment of gliomas aimed at the destruction of CSCs. CSCs population in the tissue of malignant gliomas causes their cellular heterogeneity because they have an indefinitely changeable term of viability and reproduction, accumulate different mutations over time and cause the development of gliomas resistance to the adjuvant treatment [2, 15-18].

Identification of CSCs in malignant gliomas is primarily determined by immunocytochemical detection of nestin expression as marker of NSCs $[19,20]$. At the same time, according to Singh S. K. et al. [21], $\mathrm{CD}_{133^{+}}$expression in the CSCs of malignant gliomas is a sufficient and necessary condition for the initiation of the tumor growth after the transplantation of CSCs into the brain of immunodeficient mice.

The expression of embryonic stem cells genes in glioblastoma CSCs explains their aggressive biology [22]. According to K. Mishima et al. [23], D. R. Larks et al. [24], R. H. Dahlrot et al. [25] the expression of stem markers podoplanin (PDPN), CD133 and nestin in CSCs of malignant gliomas may serve as predictors of clinical outcomes. At the same time, the prognostic significance of the signs of stem markers expression in CSCs is not recognized by number of authors [26-28].

Thus, adjusted identification and cell genesis of CSCs in brain gliomas are still completely unclear. Advanced study of CSCs biology in brain gliomas is an important area of immunocytochemical and molecular genetic studies of these tumors at the present stage.

An important role in these studies belongs to the experimental method of cell culture, which allows selecting of CSCs populations from the original tumor tissue as a part of neurospheres and tracking their intravital growth dynamics. As noted by D. R. Larks et al. [24], a renewable formation of neurospheres (tumorospheres) in culture is a fundamental property of CSCs and a determining factor, initiating the growth of tumors in brain tissue. Belonging of the cultured cells 
to CSCs is proved according to the following criteria: a) generation of clonal clusters derivatives, forming neurospheres in culture; b) self-renewal and proliferation of tumor cells; c) differentiation with reproduction of the original tumor cell phenotype; d) expression of CSCs immunocytochemical markers in neurospheric cell clusters of the cultures.

Considering the high information content of the phenomenon of NSCs and CSCs neurosphere formation in terms of culture, the studies of recent years pay much attention to the development and improvement of methods for obtaining neurospheres from gliomas tissue with differentiated identification of their cell composition.

A significant impact on the efficiency and dynamics of neurosphere formation of cultured CSCs has an initial seeding density of tumor cells. Thus, at low cell seeding density nonadherent colonial cell clusters are formed from generated NSCs microaggregates in serum-free medium with growth factors. They are called neurospheres [29]. Later it was shown that cells isolated from the children's brain tumors and glioblastoma multiforme (GBM), at low cell seeding density in serum-free media, are also capable of forming neurospheres with a high potential for proliferation and self-renewal and can exhibit potency to multilineage differentiation into neuronal and glial lines with forming a certain number of abnormal cells with a mixed phenotype [16].

At high seeding density neurospheres can aggregate and clump together [30]. Therefore, a quantitative indicator of neurosphere formation in culture should be considered as credible only at initially low seeding density of tumor cells $\left(<1 \cdot 10^{5}\right.$ cells $\left./ \mathrm{cm}^{2}\right)$ [31]. Despite these limitations, the method of neurospheres culture is considered to be a highly informative way to study the biology of malignant gliomas CSCs.

Neurospheres are heterogeneous aggregates derived from early progenitor cells or a CSCs. During dissociation of neurospheres cells of such cultures are suitable for serial plating, wherein the proportion of cells forms the secondary or tertiary neurospheres over many passages. The formation of invasive tumors is proved on models of intracerebral transplantation of such neurospheres' xenografts [21].

In experiments Qiang L. I. et al. [32] found that serum treatment of neurospheres, obtained in GBM culture, leads to an astroglial differentiation of some cells. On the other hand, it was found that certain adhesive GBM lines obtained in serum-containing medium can also form neurospheres.

Gilbert C. A. et al. [33] traditionally grew GBM cells in adhesive culture with serum, where the majority of forming neurospheres showed tumorigenic properties. However, some of these cultures, in contrast to the initial tumor patients with GBM, did not have invasive properties of tumor growth when transplanted into brains of immunodeficient mice. At the same cultured cells of neurospheres expressed high levels of differentiation markers, therefore these cultures, according to the authors, were not suitable for studying CSCs biology.

The neurospheres cells, obtained by culture of glial brain tumors in serum media, express such NSCs genes as Musashi-1 (Msi-1), Sox2, and Bmi-1 [34]. However, in serum-free nutrient media neurospheres cells reproduce gene expression profile of the original tumor more precisely than in cultures containing serum [8].

Thus, a key factor in the system of neurosphere culture is the use of serum-free medium of definite content that provides growth of CSCs in neurospheres. CSCs reproduce population of cells with the NSC-related genes and have less genetic alterations only under these conditions [33].

While culture a number of experimental glial cell lines, the individual variability in CSCs output in different lines ranged from 1-30\%, which is due to their different ability to form neurospheres. These experiments also proved that the majority of cells in the neurospheres belong to more differentiated transiently amplified cells [35].

According to observations of Lee J. C. et al. [8] activity of neurosphere formation and morphology of neurospheres in serum-free culture media change over time during 4 months of culture. Gilbert C. A. et al. [33] observed a modification of neurospheres structure of more than 20 passages from the dense clusters with almost indistinguishable individual cells to looser cell clusters.

It is generally agreed that the reproducibility of neurospheres formation of gliomas CSCs in culture conditions is affected by the timing of neurospheres dissociation. Thus, at earlier neurosphere dissociation the percentage of neurosphere-initiating cells increases and at a later dissociation of neurospheres a likelihood of cell death raises.

Among the methodological procedures affecting the efficiency of CSCs neurosphere formation, a great value is given to methods of neurospheres dissociation. The most common method for obtaining a single cell suspension is a mechanical dissociation of neurospheres using pipetting. Along with this an enzymatic dissociation of neurospheres with a number of enzymes is also applied. However, for example, the use of proteases could damage the surface of cell membranes and markers, particularly $\mathrm{CD} 133^{+}$, which leads to an underestimation of CSCs number in the cell suspension and their ineffective sorting. Nevertheless, several laboratories have successfully obtained neurospheres culture from GBM using enzymatic dissociation of the tumor tissue and cell culture in serum-free medium supplemented with growth factors [8, 24, 36-39].

The method of neurosphere $\mathrm{pH}$-dissociation is less rigid with respect to the cells than the traditional mechanical dissociation, and properties of CSCs in culture are not affected. Thus, Gilbert C. et al. [33] applied a short dissociation of neurospheres in highly alkaline medium with gentle pipetting and thereby provided effective maintenance of tumor neurospheres in the primary cultures over many passages.

The study of neurospheres structure has certain difficulties. Thus, the presence of differentiated cell descendants and occurrence of necrosis in the neurospheres resulting from the dense cell packing inhibit the diffusion of growth factor to most cells [40].

S. Facchino et al. [41] demonstrated that purified CD133+ GBM cells of the brain form neurospheres in culture, and with differentiation inducers can differentiate into neurons and glial cells. According to the authors, $\mathrm{CD} 133^{+}$cells derived from GBM, can induce very aggressive tumors when transplanted into the mice brain in vivo. Such tumors are resistant to chemotherapy and radiotherapy. They are also responsible for the development of a tumor and its continued growth after the traditional treatment of these patients with GBM.

Yi L. et al. [42] discovered that the neurospheres obtained at culture of primary human GBM as well as from a murine GBM cell line (GL261-NS), when injected into syngeneic mice brains respectively demonstrated increased tumorigenicity, forming malignant gliomas in the brain of these animals.

Tumor cells obtained from samples of human gliomas can grow as adherent cultures on laminin treated substrates in serum-free medium for neurospheres culture. The advantage of adhesive cultures is an equally efficient access of all the cells of the monolayer to growth factors preventing differentiation and apoptosis. In adherent glioma cultures CSCs are less heterogeneous in comparison to the culture of neurospheres and almost all cells express markers of CSCs - Sox2, nestin, CD133 and CD44. Only some of these cells express markers of differentiation. At intracerebral injection to immunocompetent mice only 100 cells from adherent cultures formed invasive brain tumors. In this regard, it is believed that CSCs adhesion cultures represent excellent system for screening the medicines effectiveness, as they are capable of producing adherent cell lines of all evaluated gliomas with good cell viability [6].

Pavon L. F. et al. [43] developed an optimal method for obtaining neurospheres from primary culture of human GBM with subsequent selection of $\mathrm{CD}_{133^{+}}$cells that formed subspheres from $\mathrm{CD} 133^{+}$subpopulation. In the cells of subspheres there were identified markers GFAP, CD133, nestin, Nanog, CD34, Sox2, CD44 and CD90. The authors found out that neurospheres derived from primary cultures of 
GBM contain $29 \%$ increase of cells expressing CD133 in comparison with cells from the native tumor. This means a higher concentration of $\mathrm{CD}_{133^{+}}$cells in the neurospheres formed in the primary GBM culture, in which about $89 \%$ cells express CD133 versus $60 \% \mathrm{CD}^{2} 33^{+}$cells derived from the native tumor.

Li S. C. et al. [44] addressed the issue of the signs of identity and differences of CSCs and NSCs from neuroregenerative and hippocampal regions of the subventricular zone of the brain. In analyzing relationship between the CSCs and normal NSCs a particular attention is paid to NSCs marker CD133, which is more consistently detected in cells of the brain glioma, especially in GBM. The authors found a high level of its expression in studied glioma tissues and in glioma cell lines U251 and U87MG. However, it remains unclear whether CD133-positive CSCs derive from CD133-positive normal NSCs [32]. In this regard, the development of anti-tumor therapy aimed at destroying $\mathrm{CD}_{133^{+}}$ cancer stem cells (CSCs) without damaging normal NSCs is actively discussed.

Li S. C. et al. [44] succeeded in isolating CSCs from the rare tissue of human GBM, affecting neurogenerating region of the lateral ventricle wall of the brain. Microscopically this GBM had a characteristic histological structure with marked cellular polymorphism, high mitotic activity and necrotic foci of varying size. Isolated from this GBM tissue $\mathrm{CD}^{2} 33^{+}$cells also expressed markers of CSCs: nestin, CD133, Ki67, Sox2, EFNB1, EFNB2, EFNB3, Cav-1, Musashi, Nucleostemin, Notch2, Notch4, Pax6. In serum-free cultures with the growth factors EGF and bFGF $\mathrm{CD}_{133^{+}}$cells formed neurospheres. Stem properties of these cells are confirmed by the development of malignant gliomas in the brains of immunodeficient NOD/SCID mice after stereotaxic intracerebral transplantation.

According to observations of the authors CSCs of recurrent GBM of the same patient showed higher than in the primary tumor expression of CD133 in both native tissue and culture. Isolated from this GBM $\mathrm{CD}_{133^{+}}$tumor cells proliferated in agar and formed colonies of various sizes. It was also noted that $\mathrm{CD} 133^{+}$cells adhered to the fibronectin in serum culture medium and formed neurite-like processes, represent the expression of phenotypic attribute of neurogenic differentiation, confirmed by the expression of differentiation markers GFAP and $\beta$-tubulin.

Without a common set of CSCs markers Hasselbach L. A. et al. [31] proposed the use of cells derived from the sorting of normal CSCs-enriched cultures. GBM cells, which were selected according to the ability to form neurospheres in culture medium with growth factors, expressed NSCs markers. At the same time Sox2 and nestin expression constantly appeared in neurosphere cells, whereas CD133 was present only in the neurospheres formed by GBM cells [38]

Hasselbach L. A. et al. [31] optimized a protocol of enzymatic dissociation of GBM tumor tissue on more than 100 samples. In long-term GBM cultures the efficiency in neurospheres obtaining was more than $40 \%$. These neurospheres showed tumorigenicity, multilinear differentiation potential, retained the original tumors genotype, in contrast to the cultures with $10 \%$ fetal bovine serum (FBS) and also reproduced histological structure of the original tumor after orthotopic implantation of CSCs in the brain of immunodeficient mice.

Also the study shows alternative protocols of permanent or periodic culture of tumors in serum free medium with EGF and bFGF. For the selection of cells with CSCs phenotype authors have developed a step by step protocol for obtaining of neurospheres from dissociated samples of GBM in monolayer cultures growing on surfaces coated with ECM proteins.

In another series of studies Hasselbach L. A. et al. [31] compared primary GBM cells cultured in either neurosphere medium with added growth factors, or in conventional nutrient media with $10 \%$ FBS. Isolation of CSCs from dissociated cells of GBM tumors was performed using cell sorting based on the expression of CSCs markers CD133 [21, 45]. It was found that the phenotype of CSCs is not always associated with the expression of this marker, as indicated also Beier D. et al. [46], Joo K. M. et al. [47], Son M. J. et al. [48].

V. Clement et al. [49] also believe that proposed as a CSCs marker CD133 cannot be used with sufficient specificity for the identification of tumor cells with stem properties. In this regard, the authors proposed an alternative method for isolation of a subpopulation of freshly isolated tumor cells from the tissue of human glioma and glioma cultures. It is based on phenotypic signs of glioma-initiating cells without the use of molecular markers, namely on the ability of studied cells to autofluorescence in FL1 channel. It is shown that these cells are able to regeneration in vitro and tumorigenesis in vivo and selectively express genes of stem cells. According to the authors, this method of the study of gliomas tumorigenic potential can optimize the development of new therapeutic and diagnostic approaches in the treatment of human gliomas.

Thus, in numerous studies on methods of isolation and identification of CSCs in malignant gliomas of the brain, the method of neurospheres culture became a widespread and defining approach due to the preservation of molecular features of CSCs from original GBM tissue and their tumorigenic potential [8, 16, 24, 36, 37, 39, 50-52].

Considerably interesting are the works that analyze the prognostic value of the activity of neurospheres formation in culture and the expression of CSCs markers for the clinical assessment of the malignant brain gliomas outcomes [22, 53-57].

On the basis of these studies, there was found a close relationship between clinical outcome and content of CSCs in gliomas tissue, which is confirmed by formation of tumorospheres in culture, expression of stem markers and genetic traits of CSCs [9-13, 22, 24, 53-58].

Thus, Laks D. R. et al. [24] in a retrospective study on samples of 32 gliomas in culture studied the relationship between their ability to neurospheres formation, tumorigenicity and patients' outcome. Multivariate analysis of the relationship between these indicators revealed that the activity of neurospheres formation is a stable predictor of gliomas progression, disease outcome, and increased risk of the patient's death irrespective of the Ki67 proliferation index in the original tumor tissue. The authors argue that the culture of the neurospheres is an information model for the study of malignant gliomas biology.

When culture GBM samples of 39 patients, who received postoperative chemotherapy and radiotherapy, B. H. Kong et al. [59] observed the formation of tumorospheres, in which CSCs expressed immunocytochemical markers PDPN, CD133 and nestin, and showed the ability to neurogenic differentiation and tumorigenesis induction in vivo. Analysis of the dependence of CSCs neurospheres formation level in culture and of patients' survival criterion showed statistically significant differences in overall survival among positive and negative on identifying of CSCs in patients with primary GBM. B. H. Kong et al. [59] also believe that the evaluation of GBM tumorospheres formation activity in culture is an independent predictor of clinical outcome in these patients. At the same time, the authors suggest that due to a temporal duration of tumors culture for obtaining neurospheres this method may not be suitable for predicting the outcome of patients with primary GBM with a short survival period. In such cases, it is more preferable to evaluate the expression of CSCs surface antigens in native tissue of these tumors.

Advanced study of CSCs biology acquired especially important theoretical and practical significance for improving the methods of increasing the efficiency of adjuvant therapy of brain tumors. Researches of some world's laboratories are dedicated to this purpose. It is believed that CSCs subpopulation may be a critical therapeutic target to achieve full or long response of malignant gliomas on their therapeutic treatment $[60,54,61]$

It has been established that glioma cells enriched with CSCs subpopulation show increased resistance to radiation, the main means of the standard treatment of malignant gliomas, as CSCs activate the way of the intracellular response to radiation damage of DNA, quickly 
repairing them [45]. Moreover CSCs play a key role in the formation of new blood vessels that provide a progressive and continued growth of malignant gliomas after their partial removal [62].

Earlier, K. Mishima et al. [23] and A. Ernst et al. [63] reported that the expression of PDPN proved prognostic significance in patients with astrocytomas. The expression of nestin in CSCs T. Strojnik et al. [64] and CD133 R. Pallini et al. [65] associated with poor outcomes of brain tumors as well as many cancers. However, when analyzing the relationship between the expression of specific immunocytochemical markers PDPN, CD133 and nestin in CSCs cultures and survival of patients with GBM Kong B. H. et al. [59] failed to identify distinct patterns. Thus, it appeared that the average survival time in patients with positive and negative PDPN expression in CSCs was similar, accounting for 400 and 408 days, respectively. At the same time expression of CD133 and nestin was detected in cultured CSCs of most patients. Only in one case there was found negative expression of these markers in GBM cell cultures, so the authors could not perform a statistical comparison of these two groups. Similar results were obtained by Chennai P. et al. [66].

In recent years, malignant gliomas CSCs were used for testing anticancer drugs. For this purpose, there are increasingly involved CSCs cultures of neurospheres obtained from experimental lines of malignant gliomas that are considered a conventional preclinical model for the development of new therapeutic strategies.

The most promising models are highly invasive intracranial CT$2 \mathrm{~A}$ tumors of mice derived from malignant astrocytoma induced by the intracerebral administration of 20-methylcholanthrene in C57/BL6 mice [18]. In neurospheres cultures of that line E. Binello et al. [18] confirmed the stem properties of CSCs and optimized reproducibility of the injection parameters.

E. Binello et al. [18] also find apparent involvement of CSCs in the development of human gliomas with a high degree of malignancy, resistant to the standard therapy, and found that the expression of the CSCs marker CD133 in CT-2A cultures increases from $2 \%$ in the cells of monolayer up to $31 \%$ in the cells of neurospheres. Research of CSCs markers (Oct4, Nanog and nestin) showed differences in the characteristics of the monolayer cells expressing 0ct4 and nestin (but not Nanog), and neurospheres cells expressing all three markers. Moreover, CT-2A cells showed higher proliferation and aggressiveness compared with U87 cells, and cells of CT-2A neurospheres had higher proliferative potential than the cell monolayer in vitro. In this regard, the authors propose to use this model in the preclinical testing of novel therapeutic means against human gliomas with a high degree of malignancy [18].

Burkhardt J. K. et al. [67] carried out a comparative study of the impact of a number of chemotherapeutic agents on neural stem/progenitor cells (NSCs) and gliomas CSCs on the cell lines of NSCs and CSCs in culture conditions. NSCs in comparison to CSCs are more vulnerable to the impact of temozolomide and carboplatin. The classic expression of MGMT was similar in both cell lines and does not explain the observed differences in the resistance of cells to temozolomide. At the same time, there was no difference between the CSCs and NSCs in the expression of repair enzymes MLH1 and MLH2, responsible for the development of resistance to cisplatin. However gliomas CSCs showed 10 times higher levels of ABCG2, which facilitates the removal of toxic drugs from a cell.

The authors also found that Bortezomib (BTZ) - proteasome inhibitor, and Erlotinib (ERL) - EGF tyrosine kinase inhibitor, reduced the viability of CSCs, having a minimal impact on NSCs. After the treatment of patients with BTZ there was found a significant increase in the activity of caspase-3, a key enzyme of apoptosis, in CSCs. This feature was not found in NSCs. However, unlike gliomas CSCs, proteasome activity in NSCs was increased by 5-7 times. The ERL effect on gliomas CSCs Burkhardt J. K. et al. [67] explain by much higher level of expression of the epidermal growth factor receptor (EGFR), in contrast to NSCs.

$X$. Gong et al. [68] showed that the proteasome inhibitor bortezomib (BTZ) and the growth receptor of EGF - tyrosine kinase inhibitor Erlotimib - reduce the proliferation of gliomas CSCs and do not affect the viability of NSCs. At the same time, it was found that commonly used for the treatment of gliomas Temozolimib (TMZ) and cisplatin were more toxic to normal NSCs compared to CSCs. Thus, the classical chemotherapeutic drugs used for GBM treating have various effects on NSCs and CSCs in vitro.

The main conclusion of these works is the need to find medicinal products that selectively affect gliomas CSCs, without damaging healthy brain tissue and normal NSCs [67,68]. Presented observations can initiate new research directions in the search for anticancer agents that are specific to CSCs and do not affect normal NSCs.

\section{CONCLUSION}

This review shows that, despite the large amount of accumulated information in regard to the biology of tumor stem cells, their adjusted identification and cell genesis in brain gliomas are still unclear, often contradictory and require further research. Nevertheless, the discovery of CSCs in cerebral gliomas significantly changed the views of scientists on the biology of these tumors and forced reconsidering of current strategies in the treatment of these diseases. Despite the existing differences in the literature with regard to the definitions of terminology, molecular and genetic characteristics of the CSCS and their involvement in tumorigenesis in vivo, it is an indisputable that the gliomas include CSCs subpopulations, with a high proliferative potential, determining the progression of the tumor, their therapeutic resistance to chemotherapy and radiotherapy and continued growth after surgical resection. This is confirmed by clinical observations of a short clinical remission after the combined treatment of patients with malignant gliomas with high content of CSCs. Therefore, at the current stage a depth study of CSCs biology in the brain gliomas is an important scientific direction and confirms the relevance of further research of malignant gliomas stem cell biology.

An engaged experimental method of tissue culture has made a significant contribution to the solution of this problem. It is recognized that the cell culture method provides broad opportunities for allocation of CSCs populations from the original tumors tissue as a part of neurospheres, in vivo allows to observe the dynamics of their growth over many passages and is highly informative for studying the biology of malignant gliomas CSCs. Numerous studies have shown that the renewable formation of neurospheres, (tumorospheres) in culture, is a fundamental property of tumor stem cells, which initiate the growth of tumors in the brain tissue. At the same time quantitative assessment of the activity of neurosphere formation of tumor stem cells in the dynamics of culture reflects the degree of proliferative potential and malignancy of the original tumors.

An important aspect in the study of CSCs biology is their metabolic characteristics. Method of culture, allowing to isolate a population of cells from tumor tissue, creates the possibility of genetic and immunohistochemical identification of neurospheres cell composition. Therefore it is widely used for the comparative study of CSCs obtained from brain gliomas of different histological structure and degree of anaplasia. 


\section{CONCLUSION}

The cell culture method of experimental gliomas cell lines enabled the identification of individual output fluctuations of tumor stem cells from different glial lines. This demonstrates various abilities to form neurospheres and indicates different relationships between the content of actively proliferating CSCs and more differentiated transiently amplified cells in the original tissues of these tumors.

In recent years, the culture of neurospheres, derived from tumors of human brain and experimental animals was used for testing the activity of anticancer drugs at the preclinical stage. Thanks to this research there were obtained important data for modern practical neurooncology. It turned out that the classical chemotherapeutic drugs, used for the treatment of glioblastomas, in culture conditions reveal different effects on normal NSCS and CSCS. The main conclusion of the analyzed works is the need to search for drugs that selectively affect glioma stem cells without affecting the healthy brain tissue and normal NSCS. These observations may initiate new research directions in pursuit of damaging agents, which are specific to CSCs and do not affect the normal NSCs. Analysis of the given literature results confirms the relevance of the development of new therapeutic approaches against CSCs gliomas involving the method of cell culture.

\section{REFERENCES}

1. Gursel DB, Shin BJ, Burkhardt J-K, et al. Glioblastoma Stem-Like Cells - Biology and Therapeutic Implications. Cancers. 2011; 3(2):2655-2666.

2. Park DM. Biology of Glioma Cancer Stem Cells. Mol. Cells. 2009; 28:7-12.

3. Lisyany NI. Immunologija i immunoterapija zlokachestvennyh gliom golovnogo mozga. [Immunology and Immunotherapy of malignant gliomas of the brain]. Serija «Nejroimmunologija» Kiev: Interservis - Series «Neuroimmunology». Kiev: Interservice. 2011; 5:240 p.

4. Wang JC. Evaluating Therapeutic efficacy against cancer stem cells: New challenges posed by a new paradigm. Cell Stem Cell. 2007; 1:497-501.

5. Bleau $A M$, Howard BM, Taylor $L A$, et al. New strategy for the analysis of phenotypic marker antigens in brain tumor-derived neurospheres in mice and humans Neurosurg. Focus. 2008; 24(3-4):E 28.

6. Pollard SM, Yoshikawa K, Clarke ID, et al. Glioma stem cell lines expanded in adherent culture have tumor-specific phenotypes and are suitable for chemical and genetic screens. Cell Stem Cell. 2009; 4:568-580.

7. Sanai N, Alvarez-Buylla A, Berger MS. Neural stem cells and the origin of gliomas. Engl. J. Med. 2005; 353:811-822.

8. Lee J, Kotliarova $S$, Kotliarov $Y$, et al. Tumor stem cells derived from glioblastomas cultured in bFGF and EGF more closely mirror the phenotype and genotype of primary tumors than do serum-cultured cell lines. Cancer Cell. 2006; 9:391-403.

9. Kong BH, Park N-R, Shim J-K, et al. Isolation of glioma cancer stem cells in relation to histological grades in glioma specimens. Child's Nervous System. 2013; 29(2):217-229

10. Kok M, Koornstra RH, Margarido TC, et al. Mammosphere-derived gene set predicts outcome in patients with ER-positive breast cancer. The Journal of Pathology. 2009; 218(3):316-326.

11. Kim HS, Yoo SY, Kim KT, et al. Expression of the stem cell markers CD133 and nestin in pancreatic ductal adenocarcinoma and clinical relevance. International Journal of Clinical and Experimental Pathology. 2012; 5(8):754-761.

12. Pirozzi G, Tirino V, Camerlingo R, et al. Prognostic value of cancer stem cells, epithelial-mesenchymal transition and circulating tumor cells in lung cancer. Oncology Reports. 2013; 29(5):1763-1768.

13. Bao B, Ali S, Ahmad A, et al. Differentially expressed miRNAs in cancer-stem-like cells: markers for tumor cell aggressiveness of pancreatic cancer. Stem Cells and Development. 2014; 23(16):1947-1958.

14. Wang $X$, Ren $H$, Zhao $T$, et al. Stem cell factor is a novel independent prognostic biomarker for hepatocellular carcinoma after curative resection. Carcinogenesis. 2014; 35(10):2283-2290.

15. Reya T, Morrison SJ, Clarce MF, et al. Stem cells cancer and cancer stem cells. Nature. 2001; 414:105-111.

16. Singh SK, Clarke ID, Terasaki M, et al. Identification of Cancer Stem Cell in Human Brain Tumors. Cancer Res. 2003; 63(18):5821-5828.

17. Sulman E. Aldape K, Colman H. Brain tumor stem cells. Current Problems in Cancer. 2008; 32(3):124-142.

18. Binello E, Qadeer ZA, Kothari HP, Emdad L, et al. Stemness of the CT-2A Immunocompetent Mouse Brain Tumor Model: Characterization In Vitro. J. Cancer. 2012; 3:166-174.

19. Dahlstrand J, Collins VP, LendahI U. Expression of the class VI intermediate filament nestin in human central nervous system tumors. Cancer res. 1992; 52:5334-5341.

20. Tohyama T, Lee VM, Rorke LB, et al. Nestin expression in embryonic human neuroepithelium and in human neuroepithelial tumor cells. Lab. Invest. 1992; 66:303-313.

21. Singh SK, Hawkins C, Clarke ID, et al. Identification of human brain tumor initiating cells. Nature. 2004; 432:396-401.

22. Ben-Porath I, Thomson NW, Carev V J, et al. An embryonic stem cell-like gene expression signature in poorly differentiated aggressive human tumors. Nat. Genet. 2008; 40:499-507.

23. Mishima $K$, Kato $Y$, Kaneko MK, et al. Increased expression of podoplanin in malignant astrocytic tumors as a novel molecular marker of malignant progression. Acta Neuropathologica. 2006; 111(5):483-488.

24. Laks DR, Masterman-Smith M, Visnyei K, et al. Neurosphere formation is an independent predictor of clinical outcome in malignant glioma. Stem Cells. 2009; 27(4):980-987.

25. Dahlrot RH, Hermansen SK, Hansen $S$, et al. What is the clinical value of cancer stem cell markers in gliomas? International Journal of Clinical and Experimental Pathology. 2013; 6(3):334-348.

26. Christensen K, Schrøder HD, Kristensen BW. CD133 identifies perivascular niches in grade II-IV astrocytomas. Journal of Neuro-Oncology. 2008; 90(2):157-170.

27. Kim K-J, Lee K-H, Kim H-S, et al. The presence of stem cell marker-expressing cells is not prognostically significant in glioblastomas. Neuropathology. 2011; 31(5):494-502.

28. Schoppmann SF, Berghoff AS, Jesch B, et al. Expression of podoplanin is a rare event in sporadic gastrointestinal stromal tumors and does not influence prognosis. Future Oncology. 2012; 8(7):859-866.

29. Reynolds BA, Tetzlaff W, Weiss S. A multipotent EGF-responsive striatal embryonic progenitor cell produces neurons and astrocytes .J. Neurosci. 1992; 12:4565-4574.

30. Singec I, Knoth R, Meyer RP, et al. Defining the actual sensitivity and specificity of the neurosphere assay in stem cell biology. Nat. Methods. 2006; 3:801-806.

31. Hasselbach LA, Irtenkauf SM, Lemke NW, et al. Optimization of High Grade Glioma Cell Culture from Surgical Specimens for Use in Clinically Relevant Animal Models and 3D Immunochemistry. J. Vis. Exp. 2014; 83(e51088):1-9. 
32. Qiang L, Yang Y, Ma YJ, et al. Isolation and characterization of cancer stem like cells in human glioblastoma cell lines. Cancer Lett. 2009; 279(1):13-21.

33. Gilbert CA, Ross AH. Cancer Stem cells: Cell Culture, Markers and Targets for New Therapies. J.Cell.Biochem. 2009; 108(5):1031-1038.

34. Hemmati HD, Nakano I, Lazareff JA, et al. Cancerous stem cells can arise from pediatric brain tumors. Proc. Natl. Acad .Sci. USA. 2003; 100:15178-15183.

35. Ahmed S. The culture of neural stem cells. J. Cell Biochem. 2009; 106:1-6.

36. Galli R, Binda E, Orfanelli U, et al. Isolation and characterization of tumorigenic, stem-like neural precursors from human glioblastoma. Cancer Res. 2004; 64(19): 7011-7021.

37. Vescovi AL, Galli R, Reynolds BA. Brain tumour stem cells. Nat. Rev. Cancer. 2006; 6:425-436.

38. deCarvalho AC, Nelson K, Lemke N, et al. Gliosarcoma Stem Cells Undergo Glial and Mesenchymal Differentiation In Vivo. Stem Cells. 2010; 28(2):181-190.

39. Azari H, Millette S, Ansari S, et al. Isolation and expansion of human glioblastoma multiforme tumor cells using the neurosphere assay. J. Vis. Exp. 2011: 56(e3633):1-5.

40. Woolard K, Fine HA. Glioma stem cells: better flat than round. Cell Stem Cell. 2009; 4: 466-467.

41. Facchino $S$, Abdouh $M$, Chatoo $W$, et al. BMl1 confers radioresistance to normal and cancerous neural stem cells through recruitment of the DNA damage response machinery. J.Neurosci. 2010; 28(30):1096-1111.

42. Yi L, Zhou C, Wang B, et al. Implantation of GL261 neurospheres into C57/BL6 mice: a more reliable syngenic graft model for research on glioma-initiating cells. Int.J.Oncol. 2013; 43(2): 477-484.

43. Pavon $L F$, Marti $L C$, Sibov TT, et al. In vitro analysis of neurospheres derived from glioblastoma primary culture: a novel methodology paradigm. Front.Neurol. 2014; 4:214-220.

44. Li SC, Long TV, Ho HW, et al. Cancer stem cells from a rare form of glioblastoma multiforme involving the neurogenic ventricular wall. Cancer Cell International. 2012; 12:41-60.

45. Bao S, Wu Q, MCLendon DE, et al. Glioma stem cells promote radioresistance by preferential activation of the DNA damage response. Nature. 2006a; 444:756-760.

46. Beier D, Hau P, Proescholdt M, et al. CD133(+) and CD1339(-) glioblastoma-derived cancer stem cell show differential growth characteristics and molecular profiles. Cancer Res. 2007; 67: 4010-4015.

47. Joo KM, Kim SY, Jin X, et al. Clinical and biological implications of CD133-positive and CD133-negative cells in glioblastomas. Lab. Invest. 2008; 88:808-815.

48. Son MJ, Woolard K, Nam DH, et al. SSEA-1 is an enrichment marker for tumor-initiating cells in human glioblastoma. Cell Stem cell. 2009; 4:440-452.

49. Clement V, Marino D, Cudalbu C, et al. Marker-independent identification of glioma-initiating cells. Nature Methods. 2010; 7:224-228.

50. Ignatova TN, Kukekov VG, Laywell ED, et al. Human cortical glial tumors contain neural stem-like cells expressing astroglial and neuronal markers in vitro. Glia. 2002; 39(3):193-206.

51. Yuan X, Curtin J, Xion Y, et al. Isolation of cancer stem cells from adult glioblastoma multiforme. Oncogene. 2004; 23:9392-9400.

52. Germano IM, Swiss V, Casaccia P. Primary brain tumors, neural stem cells, and brain tumor cancer cells: Where is the link? Neuropharmacol. 2010; 58(6):903-910.

53. Strojnik T, Røsland GV, Sakariassen PO, et al. Neural stem cell markers, nestin and musashi proteins, in the progression of human glioma: correlation of nestin with prognosis of patient survival. Surgical Neurology. 2007; 68(2):133-143.

54. Beier D, Rohrl S, Pillai DR, et al. Temozolomide preferentially depletes cancer stem cells in glioblastoma. Cancer Res. 2008; 68:5706-5715.

55. Pallini R, Ricci-Vitiani L, Banna GL, et al. Cancer stem cell analysis and clinical outcome in patients with glioblastoma multiforme. Clinical Cancer Research. 2008; 14(24):8205-8212.

56. Zeppernick F, Ahmadi R, Campos B, et al. Stem cell marker CD133 affects clinical outcome in glioma patients. Clin Cancer Res. 2008; 14(1):123-129.

57. Zhang M, Song $T$, Yang $L$, et al. Nestin and CD133: valuable stem cell-specific markers for determining clinical outcome of glioma patients. J Exp Clin Cancer Res. 2008; 27: 85-92.

58. Wang X, Ren H, Zhao T, et al. Stem cell factor is a novel independent prognostic biomarker for hepatocellular carcinoma after curative resection. Carcinogenesis. 2014; 35(10):2283-2290.

59. Kong BH, Moon JH, Huh UM, et al. Prognostic Value of Glioma Cancer Stem Cell Isolation in Survival of Primary Glioblastoma Patients. Stem Cells International. 2014; Article ID 838950. $6 \mathrm{p}$.

60. S. Bao, Q. Wu, Z. Li, et al. Rich Targeting cancer stem cells through L1CAM supresses glioma grouth. Cancer Res. 2008; 68:7043-6048.

61. Dietrich J, Imitola J, Kesari S. Mechanisms of Disease: the role of stem cells in the biology and treatment of gliomas . Nat.Clin.Pract. 2008; 5:393-404.

62. Bao S, Wu Q, Sathornsumetae $S$, et al. Stem cell-like glioma cells promote tumor angiogenesis through vascular endothelial growth factor. Cancer.Res. 2006b; 66:7843-7848.

63. Ernst A, Hofmann S, Ahmadi R, et al. Genomic and expression profiling of glioblastoma stem cell-like spheroid cultures identifies novel tumor-relevant genes associated with survival. Clinical Cancer Research. 2009; 15(21):6541-6550.

64. Strojnik T, Rosland GV, Sakariassen PO, et al. Neural stem cell markers, nestin and musashi proteins, in the progress of human glioma: correlation of nestin with prognosis of parient survival. Surg Neurol. 2007; 68(2):133-144.

65. Pallini R, Ricci-Vitiani L, Banna GL, et al. Cancer stem cell analysis and clinical outcome in patients with glioblastoma multiforme. Clin Cancer Res. 2008; 14(24):82058212.

66. Chinnaiyan $P$, Wang $M$, Rojiani AM, et al. The prognostic value of nestin expression in newly diagnosed glioblastoma: report from the radiation therapy oncology group. Radiation Oncology. 2008; 3(1):32-40.

67. Burkhardt JK, Shin BJ, Boockvar JA. Neural Stem Cells and Glioma Stem-Like Cells Respond Differently to Chemotherapeutic Drugs: Selectivity at the Cellular Level. Neurosurgery. 2011; 68(6):21-22.

68. Gong X, Schwartz PH, Linskey ME, et al. Neural stem/progenitors and glioma stem-like cells have differential sensitivity to chemotherapy. Neurology. 2011; 76(13):1126-1134.

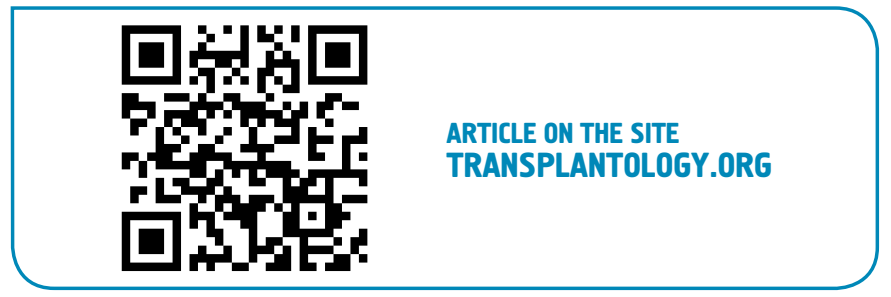

The authors indicate no potential conflicts of interest. 